\title{
The NeQuick model genesis, uses and evolution
}

\author{
Sandro M. Radicella \\ ARPL, The Abdus Salam ICTP, Trieste, Italy
}

\begin{abstract}
The genesis and evolution of the NeQuick model is reviewed from the initial ionospheric efforts made in the framework of the European COST actions on ionospheric issues to the last version of the model (NeQuick 2). Attention is given to the uses of the model particularly by the European satellite navigation and positioning systems EGNOS and GALILEO. Recent assessment studies on the performance of NeQuick 2 are also reviewed.
\end{abstract}

Key words ionospheric model-model assessment - model uses

\section{Introduction}

The NeQuick ionospheric model is based on a model (DGR from now on) introduced by Di Giovanni and Radicella (1990).

The original DGR model uses a sum of Epstein layers to reproduce the electron density distribution in the ionosphere analytically. Its formulation is such that the function and its 1st derivative are always continuous. The construction of the analytical function is based on «anchor» points related to the ionospheric characteristics routinely scaled from ionograms $(f o F 2$, $M(3000) F 2, f o F 1, f o E)$. For this reason the DGR model is essentially a «profiler». The original DGR «profiler» can be used with experimental or modeled data of the ionospheric characteristics.

An improved version of the original DGR introduced by Radicella and Zhang (1995) de-

Mailing address: Dr. Sandro M. Radicella, ARPL, The Abdus Salam ICTP, Strada Costiera 11, 34151 Trieste, Italy: e-mail: rsandro@ictp.it scribes the topside $F 2$ region by introducing a constant shape factor $k$ that modifies the thickness parameter for that region and can be derived empirically by comparison with experimental vertical TEC data. The improved version of the DGR profiler calculates vertical TEC values.

The modeling effort described above was part of the activities done under the scheme of COST 238 (Prediction and Retrospective Ionospheric Modeling over Europe, PRIME). The improved DGR «profiler» was adopted by the action as part of its final product.

A new family of electron density models, differing in complexity and whith different but related application areas, based on the DGR «profiler» concept has been developed in collaboration with the University of Graz, Austria.

These models are particularly suited for the study of trans-ionospheric radiopropagation effects of interest to satellite navigation and positioning (Hochegger et al., 2000; Radicella and Leitinger, 2001 ).

The models are:

- NeQuick - a quick-run model for transionospheric applications;

- COSTprof - a model suited for ionospheric and plasmaspheric satellite to ground applications; 
- NeUoG-plas - a model suited for assessment studies involving satellite-to satellite propagation of radio waves.

All three models give electron density as a function of height, geographic latitude, geographic longitude, solar activity (specified by the sunspot number or by the $10.7 \mathrm{~cm}$ solar radio flux), season (month) and time (Universal Time UT or local time LT). The models, like the original DGR, are continuous in all spatial first derivatives, particularly needed in applications like ray tracing and location finding. They also allow the calculation of electron density along arbitrarily chosen ray paths and the corresponding total electron content (TEC). COST prof model was adopted in the final product of COST 251 action (Improved Quality of Service in Ionospheric Telecommunication Systems Planning and Operation).

\subsection{The NeQuick model and its uses}

Particularly successful was the development of the NeQuick model. To describe the electron density of the ionosphere above $100 \mathrm{~km}$ and up to the $F 2$ layer peak this model uses a modified DGR profile formulation. A semi-Epstein layer represents the electron density distribution in the topside with a height dependent thickness parameter empirically determined.

The model has been adopted by the International Telecommunication Union, Radiocommunication Sector (ITU-R) Recommendation P. 531-6, now superseded by P. 531-9, (ITU, 2007) as a suitable method for TEC modeling. The NeQuick (Fortran 77) source code is available at: http://www.itu.int/ITU-R/software/studygroups/rsg3/databanks/ionosph/. The basic inputs of the NeQuick model code are: position, time and solar flux (or sunspot number); the output is the electron concentration at the given location in space and time. In addition the NeQuick package includes specific routines to evaluate the electron density along any ray-path and the corresponding TEC by numerical integration.

The original version of the NeQuick model has been used by the European Geostationary Navigation Overlay Service (EGNOS) of the
European Space Agency (ESA) for system assessment analysis. For such objective a series of ionospheric scenarios have been created under ESA contracts to simulate realistic ionospheric conditions under disturbed conditions. These scenarios were done in collaboration with the University of Graz. Some of them were done also in collaboration with the Rutherford-Appleton Laboratory of the UK.

A series of additional uses of the original version of the model are known. It has been adapted by the Rutherford-Appleton Laboratory of the UK to forecast vertical TEC from forecasted values of $f o F 2$ and MUF(3000)F2 (Cander, 2003). It has been integrated also in the simulation toolkit developed in Australia to conduct a qualitative assessment of the performance characteristics of the future GNSS infrastructure (Seynat et al., 2004). The model is used to calculate ionospheric delays in the Raw Data Generation (RDG) capability of the Galileo System Simulation Facility (GSSF) (Zimmerman et al., 2005). It was implemented in the Global Ionospheric Scintillation Model (GISM) to calculate the background ionosphere (Beniguel and IEEA, 2004).

A very important use of the NeQuick model is, without doubt, its adoption as the model for ionospheric corrections in the single frequency operation of the European GALILEO satellite navigation system (Arbesser-Rastburg, 2006).

For its utilization by the GALILEO system the model will be driven by an «effective ionisation level» $\mathrm{Az}$, defined as follows:

$$
\mathrm{Az}(\mu)=a_{0}+a_{1} \mu+a_{2} \mu^{2}
$$

where $\mu$ is the modip, a geomagnetic coordinate introduced by Rawer (1983), and the coefficients $a_{0}, a_{1}, a_{2}$ are broadcast to the user to allow Az calculation at any wanted location.

At system level, a set of worldwide distributed monitoring stations will be used to evaluate the slant TEC needed to compute the values of the world wide parameters $a_{0}, a_{1}, a_{2}$ which describe the global behavior of $\mathrm{Az}$ for a given day.

NeQuick model driven by the parameters broadcast during the following day is applied at user level to compute the slant TEC along any ray-path satellite-receiver. 


\section{NeQuick recent evolution}

The performance of the NeQuick model has been tested intensively and a series of improvements have been introduced in its original version.

In «mapping» applications of NeQuick (construction of electron density grids at fixed heights below the $F 2$ peak) strong gradients and strange structures appear at $E$ and $F 1$-layer heights in some cases. To eliminate these problems an elaborate revision of the original DGR modeling approach for the $F 1$ and $E$ regions was necessary (Leitinger et al., 2005).

The ionosphere topside profile in the original version of the NeQuick model is based on an empirically derived thickness parameter. Two formulas are used, each of them valid for six months of the year. These formulae involve $F 2$ peak parameters, bottom-side thickness and solar activity and were given in Radicella and Zhang (1995). To estimate empirically the thickness parameter formulation for the topside a large series of TEC data derived from geostationary satellite radio signals recorded at Florence and ionosonde data from Rome were used in that early work. The possibility to derive the thickness parameter empirical formulation using newly available topside sounder data from the ISIS 2 satellite allowed introducing a substantial improvement in the topside profile of NeQuick. The profile is now characterized by a single empirical formula for the thickness parameter. Such an improvement had important consequences al- so for the evolution of the International Reference Ionosphere (IRI) electron density model. Taking into account an outcome like that indicated in Coïsson et al. (2006) and results like those shown in fig. 1 the IRI in its last version, IRI2007, adopted the NeQuick new formulation as the default option for its topside (http://omniweb.gsfc.nasa.gov/vitmo/iri_vitmo.html). Bilitza and Reinisch (2008) consider the new NeQuick topside electron density profile «the most mature of the different proposals for the IRI topside».

The improvements to the NeQuick model mentioned above and made during the COST 296 action, plus others related to the structure of the computational code itself, made possible to issue a new version of it: NeQuick 2. A full description of this version of the model and of the main differences with the previous version is given in Nava et al. (2008). Figure 2 shows an example of the electron density profile along the ray slant produced by the NeQuick 2 model. On-line NeQuick 2 calculation tool is available for demonstration purposes (http://arpl.ictp.trieste.it/nq-online/index.html).

A detailed study done under contract with the European GNSS Supervisory Authority (GSA) through France Developpement Conseil, have shown that, for GNSS single frequency operation, NeQuick in its two versions performs better than the equivalent ICA model used for GPS operations.

Parameters $a_{0}, a_{1}, a_{2}$ for a given day were computed from experimental slant TEC values obtained in a set of worldwide distributed
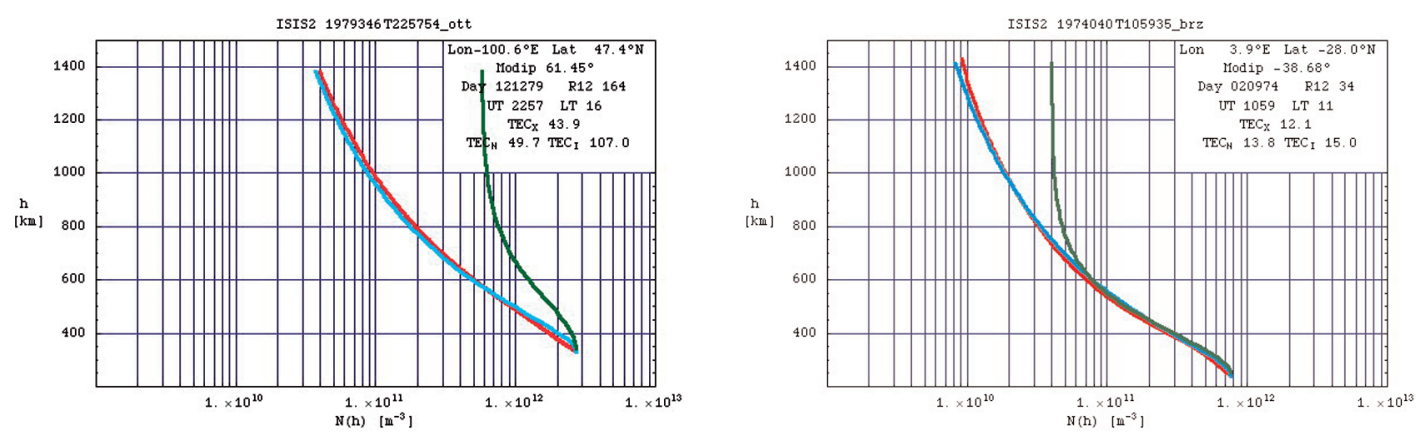

Fig. 1. Examples of comparisons among electron density topside profiles. Red curves correspond to ISIS 2 experimentally derived profiles. Green corresponds to IRI-2000 profiles and light-blue are NeQuick 2 profiles. 


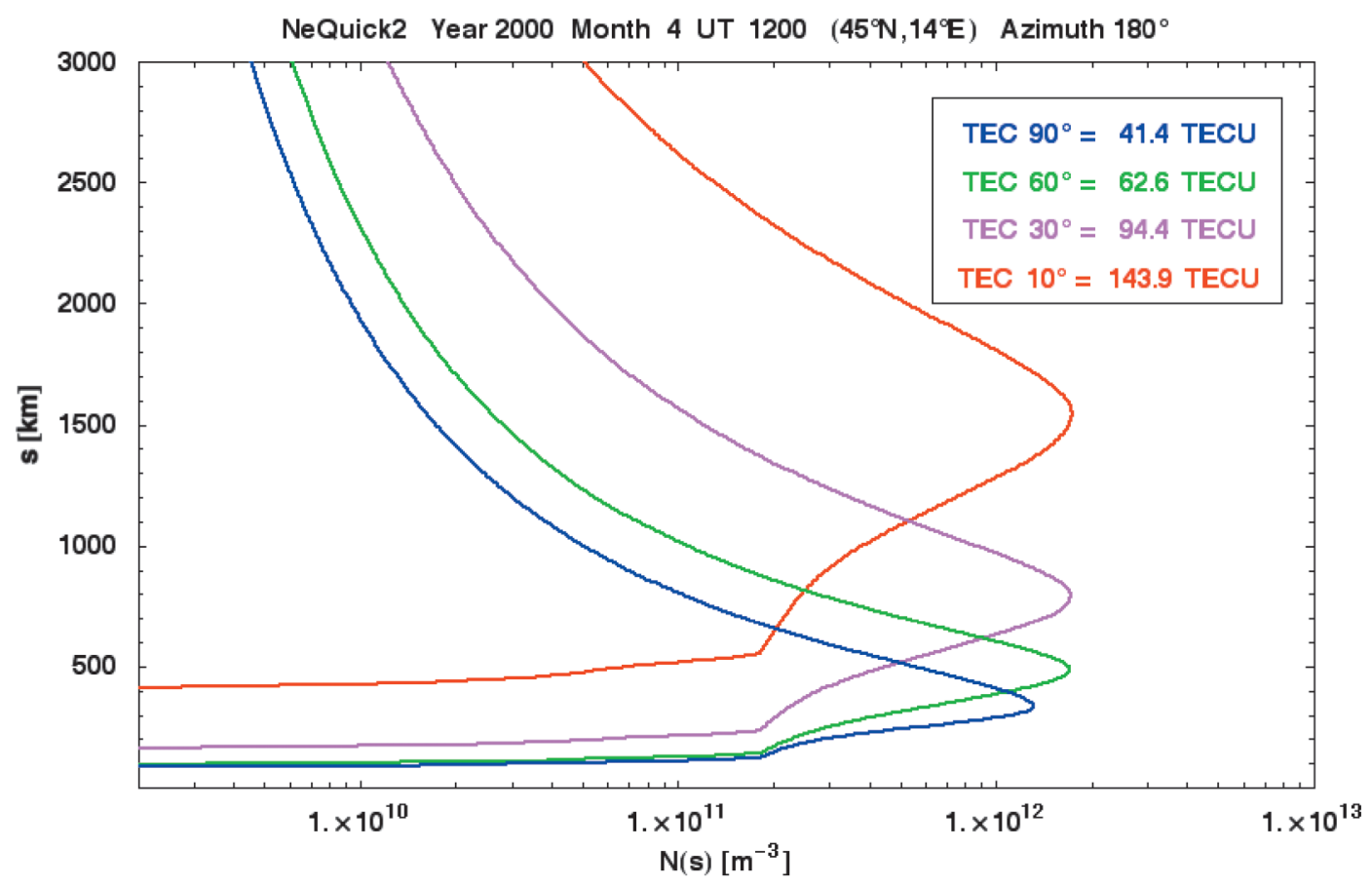

Fig. 2. Example of NeQuick 2 profiles and TEC along ray-paths. Different colors correspond to different path elevation angles. $90^{\circ}$ means vertical profile and TEC.

«monitoring» stations. These parameters were used during the following day to drive the NeQuick model and compute the slant TEC along any satellite to receiver ray-path. A specific NeQuick program adaptation was devised for that purpose.

In the case of ICA, the GPS broadcast coefficients have been used to compute the slant TEC on the same satellite to receiver links.

To perform a global analysis of the model's performance, a set of 27 test stations homogeneously distributed around the World has been chosen to cover all modip regions.

The statistical analysis was performed through cumulative distributions of the absolute values of the mis-modelling, defined as the difference between an experimental and its corresponding modelled slant TEC. It appears that in almost all cases NeQuick mis-modelling are smaller than the ICA ones, reflecting the more realistic global behavior of NeQuick. Figure 3 shows the daily values of the 95 percentile of the mis-modelling for the NeQuick 2 and the ICA models from the cumulative distribution for the year 2000 of high solar activity. Each daily value corresponds to the full set of 27 test stations.

\section{Independent evaluation of NeQuick 2 performance}

NeQuick 2 code has been requested by a large number of researchers from all over the world and its performance is being evaluated. A few examples of these evaluations follow.

Bidaine and Warnant (2008) made done a comparative study of the two versions of the NeQuick model using high solar activity data from three ionospheric soundings stations in 


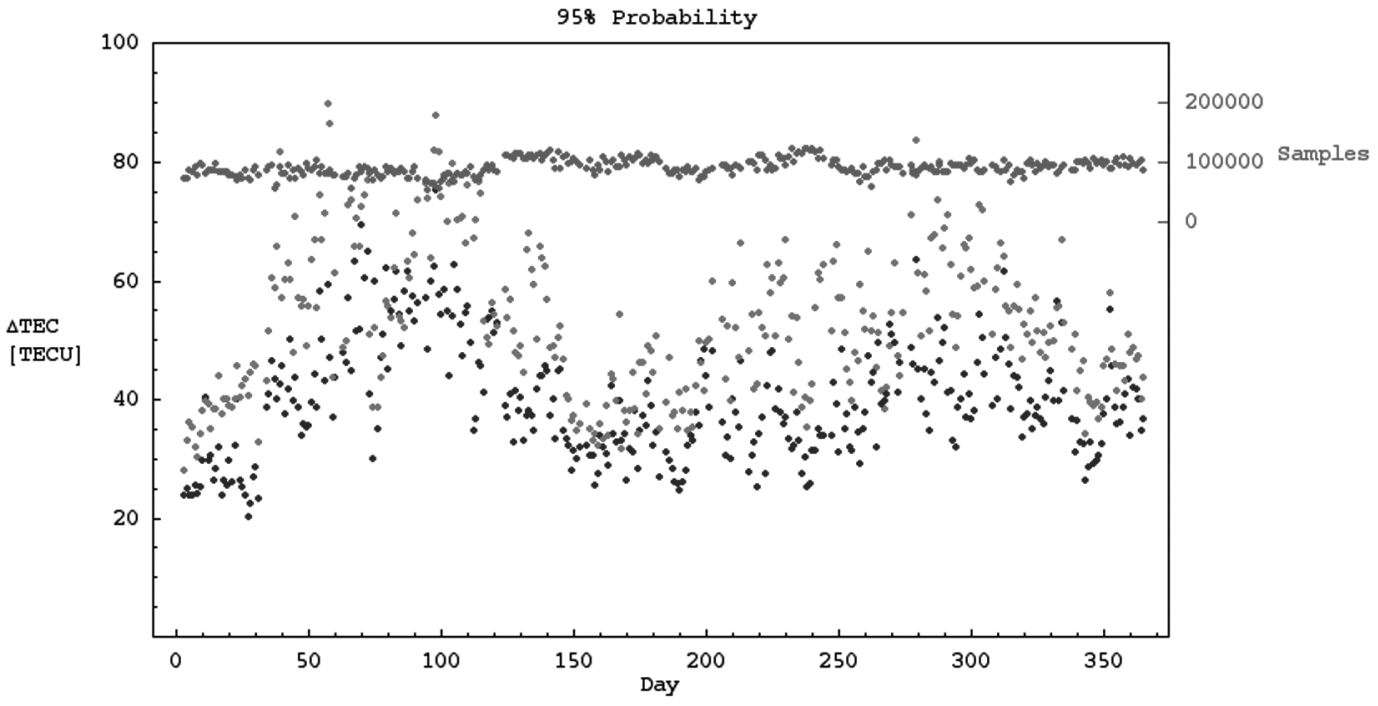

Fig. 3. 95 percentile (95\% probability) of the cumulative distribution for global daily mis-modelling (difference between an experimental and its corresponding modelled slant TEC) for the ICA model (gray) and NeQuick 2 model (black). In the top part of the figure the corresponding samples used in each daily calculation is given.

Europe (Dourbes, Roquetes and El Arenosillo) and vertical TEC derived from GPS receivers located near those stations. They concluded that the new formulation of the topside introduced in NeQuick 2 give rise to a clear homogenization of the statistics and to an improvement of the performance of the model. The paper shows that using the data obtained from the ionospheric soundings about $75 \%$ of total vertical TEC correspond to the topside of the ionosphere, making evident the importance of the improvement in the topside formulation of the model.

Orus et al. (2007) analyzed the performance of the two versions of the NeQuick model when it is used in the GALILEO single frequency operations algorithm for ionospheric corrections. These authors assessed the model performance on a global scale for the whole year 2000 using different sets of experimental data for comparison. They concluded that the NeQuick 2 version of the model improves the mis-modelling in almost all latitudes. Moreover, the new NeQuick version presents major reduction of the mis-modelling global bias, which leaves the bias for the whole year 2000 below 1 TECU (1 TECU $=10^{16} \mathrm{~m}^{2}$ ).

In preparation for the deployment of the Galileo System, the European Space Agency (ESA) initiated the development of an overall Galileo System Test Bed (GSTB-V2) including two Galileo In-Orbit Validation Element (GIOVE) satellites: GIOVE-A (launched on 28th December 2005) and GIOVE-B (launched 27th April 2008) with several objectives including ionospheric issues aspects. In this framework, Orus and Prieto-Cerdeira (2008) made an assessment study of the GALILEO single frequency algorithms based on the NeQuick model using data from the GIOVE-A experimentation campaign.

The authors concluded that NeQuick 2 version of the model improves the ionospheric corrections calculation speed (due to the improvements on the computer code of the model) and reduces some of the mis-modelling in the topside ionosphere indicating again the value of the improvements introduced in the topside model formulation. 


\section{Conclusions}

The groups of the Abdus Salam ICTP Aeronomy and Radiopropagation Laboratory and the University of Graz Institute of Geophysics, Astrophysics and Meteorology have made important ionosphere modeling efforts in the framework of different ionospheric related European COST actions and specifically in COST 296 action. One of these efforts, NeQuick model, is at present widely recognized and used internationally. In particular, it has been utilized for assessment studies by the European EGNOS project and is also adopted in the single frequency operation algorithm for ionospheric corrections for the European GALILEO system for satellite navigation and positioning.

\section{REFERENCES}

ARbeSser-RASTBURG, B. (2006): The GALILEO single frequency ionospheric correction algorithm, (3rd European Space Weather Week, Brussels).

BenIGUEL, Y. and IEEA (2004): Global Ionospheric Scintillation Model (GISM) Technical Manual, v 5.1, ITU-R RP 257 2004-10-03.

Bidaine, B. and R. WARnANT (2008): Modelling the Ionosphere over Europe: Investigation of NeQuick Formulation, presented at the $5^{\circ}$ European Space Weather Week, (Brussels).

BilitZA, D. and B. ReINISCH (2008): International Reference Ionosphere 2007: Improvements and new parameters, Adv. Space Res., 42 (4), 599-609.

CANDER, LJ. R. (2003): Towards forecasting and mapping ionospheric space weather under COST actions, $A d v$. Space Res., 31 (4), 957-964.
CoÏsson, P., S.M. Radicella, R. Leitinger and B. NAVA (2006): Topside electron density in IRI and NeQuick: features and limitations, Adv. Space Res., 37, 937-942.

Di Giovanni, G. and S.M. Radicella (1990): An Analytical Model of the Electron Density Profile in the Ionosphere, Adv. Space Res., 10 (11), 27-30.

HoCHEGGER, G., B. NAVA, S.M. RADICELLA and R. LEITINGER (2000): A Family of Ionospheric Models for Different Uses, Physics And Chemistry Of The Earth, Part C: Solar, Terrestrial \& Planetary Science, 25 (4), 307-310.

ITU-R (2007): Recommendation ITU-R P 531-9.

Leitinger, R., M.L. Zhang and S.M. Radicella (2005): An improved bottomside for the ionospheric electron density model NeQuick, Annals of Geophysics, 48 (3), 525-534.

NAva, B., P. CoÏsson and S.M. Radicella (2008): A new version of the NeQuick ionosphere electron density model, Journal of Atmospheric and Solar-Terrestrial Physics, doi:10.1016/j.jastp.2008.01.015.

Orus, R., B. Arbesser-Rastburg, R. Prieto-Cerdeira, M. Hernandez-Pajares, J.M. Juan and J. Sanz (2007): Performance of different Ionospheric models for single frequency Navigation receivers, IBSS-07 Proceedings, (Boston, 2007).

Orus, R. and R. Prieto-Cerdeira (2008): GIOVE-A Experimentation campaign: ionospheric related analysis, presented at NAVITEC, (Noordwijk).

RAdicella, S.M. and M.L. Zhang (1995): The improved DGR analytical model of electron density height profile and total electron content in the ionosphere, Annali di Geofisica, XXXVIII (1), 35-41.

Radicella, S.M. and R. Leitinger (2001): The Evolution of the DGR Approach to Model Electron Density Profiles, Adv. Space Res., 27 (1), 35-40.

Seynat, C., A. Kealy and K. Zhang (2004): A Performance Analysis of Future Global Navigation Satellite Systems, Journal of Global Positioning Systems, 3 (12), 232-241.

Zimmermann, F., T. HaAk, E. Steindl, S. Vardarajulu and O. KALDEN (2005): Generating Galileo Raw Data - Approach and Application, presented at Data Systems in Aerospace (DASIA), (Edinburgh, Scotland). 